\title{
Observer-Based Strategies for Anesthesia Drug Concentration Estimation
}

\author{
Jin-Oh Hahn¹, Guy A. Dumont ${ }^{2}$ and J. Mark Ansermino² \\ 1 University of Alberta, \\ ${ }^{2}$ University of British Columbia \\ Canada
}

\section{Introduction}

Knowledge of the anesthesia drug concentration in a patient during a surgical procedure has potential to improve patient monitoring and control of drug administration. High-fidelity estimates of anesthesia drug concentration would allow anesthesiologists to make relevant low-level dose adjustment as well as high-level clinical decisions. The estimated drug concentrations may also be useful for computerized anesthesia delivery, including, but not limited to, targeting/limiting the drug concentration itself (Van Poucke et al., 2004; Gentilini et al., 2002) as well as monitoring and maintaining hemodynamic stability (Rao et al., 2000).

Currently, anesthesia drug concentration is estimated based primarily on a model-based open-loop prediction (Fig. 1(a)), in which the drug concentration is predicted by solving a population-based patient pharmacokinetics (PK) and pharmacodynamics (PD) described in terms of a multi-compartmental model. In the absence of any feedback correction capability, the discrepancy between the real PK of an individual patient and its population-based model counterpart is a major source of errors in drug concentration estimation. The openloop prediction has been widely used despite this apparent drawback, mainly due to the lack of alternative solutions.

Recently, efforts have been made to improve the fidelity of drug concentration estimation over open-loop prediction. Sartori et al. (2005) proposed a method based on an extended Kalman filter to adapt PD parameters in real-time. Hahn et al. (2011) proposed a robust estimation strategy based on $\mathrm{H}_{\infty}$ control theory. The results strongly suggest that the efficacy of drug concentration estimation can be improved by exploiting the measurements of the clinical effect. Motivated by these pioneering investigations, this chapter aims to study alternative observer design strategies for estimating anesthesia drug concentration. In contrast to open-loop prediction, observer-based approaches can be regarded as closed-loop estimation that exploits the clinical effect measurement for feedback correction (Fig. 1(b)), in order to suppress the adverse influence of patient variability and effectively deal with unknown surgical stimulation (shown as d in Fig. 1) that acts as a disturbance to distort the clinical effect measurement. The specific focus of this chapter is to examine the design and analysis of a robust linear observer (RLO), a robust nonlinear observer (RNO), and an $\mathrm{H}_{\infty}$ observer $\left(\mathrm{H}_{\infty} \mathrm{O}\right)$ in the context of estimating propofol concentrations at the plasma and the

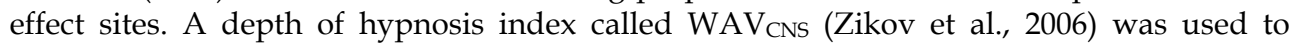


describe the clinical effect of interest for feedback correction. The observers were evaluated against the traditional open-loop prediction using a Monte-Carlo simulation of surgical procedures using a wide range of patient models. $\mathrm{H}_{\infty} \mathrm{O}$ boasted the best overall performance with its capacity to deliver statistically significant reduction in plasma and effect site propofol concentration errors over open-loop prediction, regardless of the presence of a surgical stimulation disturbance. RNO was more effective than open-loop prediction in suppressing the effect site propofol concentration error, but its efficacy for plasma propofol concentration was susceptible to surgical stimulation disturbance. RLO was good at reducing the effect site propofol concentration error in the absence of surgical stimulation disturbance, but perfomed poorly in the presence of a surgical stimulation disturbance.

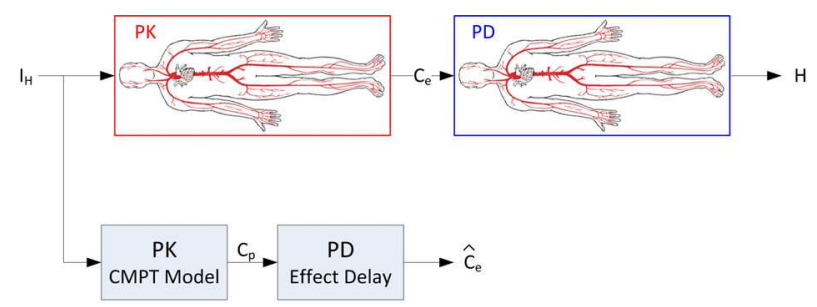

(a) Open-Loop Prediction

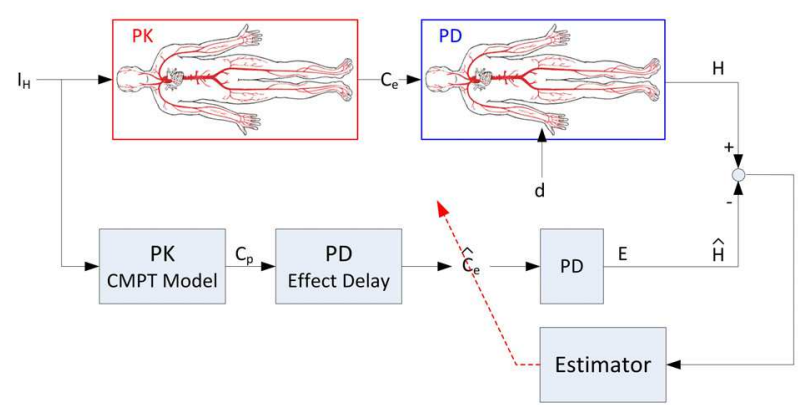

(b) Closed-Loop Estimation

Fig. 1. Open-loop prediction versus closed-loop estimation of anesthesia drug concentration.

This chapter is organized as follows. Section II describes the patient model. Section III details the design of observers for anesthesia drug concentration estimation. Section IV details the Monte-Carlo simulation and statistical analysis to compare the performance of observer-based estimation and open-loop prediction strategies. The results are presented and discussed in Section V, followed by conclusions in Section VI.

\section{Patient model}

An anesthetized patient can be described by a series connection of a PK model, a PD model, and a monitor model, as shown in Fig. 2. The input to this process is the drug infusion rate $\left(\mathrm{I}_{\mathrm{H}}\right)$, while the output is the clinical effect measurement (WAV $\left.\mathrm{CNS}_{\mathrm{C}}\right)$. The surgical stimulation is modeled as an output disturbance, because it counteracts the effect of anesthesia drug in an unpredictable manner and thereby distorts the $\mathrm{WAV}_{\mathrm{CNS}}$ measurement. 


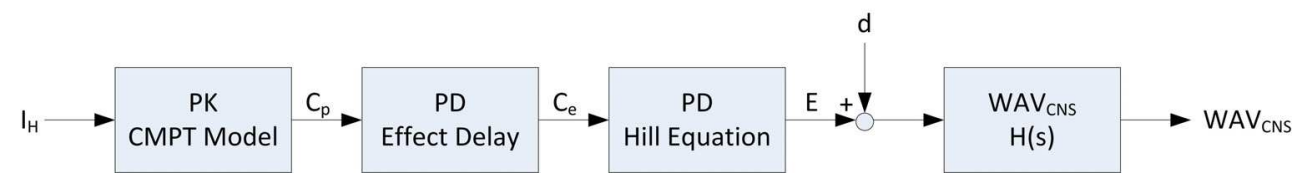

Fig. 2. PK/PD and effect monitor models of anesthesia drug delivery process.

\subsection{PK and PD models}

A 3-compartment model of propofol developed by Schüttler and Ihmsen (2000) was used to describe the PK (Fig. 3). Denoting $x_{1}, x_{2}$, and $x_{3}$ as drug concentrations in the plasma, fast peripheral, and slow peripheral compartments, the state-space representation of the PK is given by (1):

$$
\begin{aligned}
\dot{\mathbf{x}}_{\mathrm{PK}} & =\left[\begin{array}{ccc}
-\left(k_{10}+k_{12}+k_{13}\right) & k_{12} & k_{13} \\
k_{21} & -k_{21} & 0 \\
k_{31} & 0 & -k_{31}
\end{array}\right] \mathbf{x}_{\mathrm{PK}}+\left[\begin{array}{c}
V_{1}^{-1} \\
0 \\
0
\end{array}\right] u=\mathbf{A}_{\mathrm{PK}} \mathbf{x}_{\mathrm{PK}}+\mathbf{B}_{\mathrm{PK}} u, \\
C_{p} & =x_{1}=\left[\begin{array}{lll}
1 & 0 & 0
\end{array}\right] \mathbf{x}_{\mathrm{PK}}=\mathbf{C}_{\mathrm{PK}} \mathbf{x}_{\mathrm{PK}}
\end{aligned}
$$

where $\mathbf{x}_{\mathrm{PK}}=\left[\begin{array}{lll}\mathrm{x}_{1} & \mathrm{x}_{2} & \mathrm{x}_{3}\end{array}\right]^{\mathrm{T}}, \mathrm{u}=\mathrm{I}_{\mathrm{H}}, \mathrm{k}_{\mathrm{ij}}$ are the rate constants, and $\mathrm{V}_{1}$ is the volume of the plasma compartment. To account for the PD lag associated with the distribution of drug into the effect site (i.e. the brain), the following delay-plus-first-order model was used (Zikov et al., 2006):

$$
x_{4}(s)=C_{e}(s)=e^{-T_{d} s} \frac{k_{d}}{s+k_{d}} C_{p}(s)
$$

where $T_{d}$ and $k_{d}$ are respectively the transport delay and the rate of drug distribution from the plasma to the brain. Finally, the saturating Hill equation was used to describe the relation between $\mathrm{C}_{\mathrm{e}}$ and the anesthesia effect:

$$
E\left(\bar{C}_{e}\right)=\frac{\left[\bar{C}_{e}\right]^{\gamma}}{1+\left[\bar{C}_{e}\right]^{\gamma}},
$$

where $\bar{C}_{e}=\frac{C_{e}}{E C_{50}}$ is the effect site drug concentration normalized by the $50 \%$ effect concentration $\left(\mathrm{EC}_{50}\right)$, and $\gamma$ is the cooperativity coefficient.

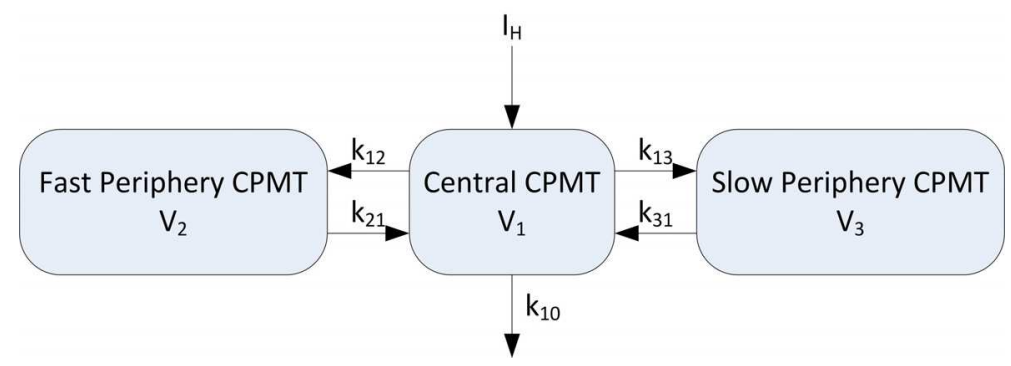

Fig. 3. A 3-compartmental PK model. 


\section{2 $\mathrm{WAV}_{\mathrm{CNS}}$ monitor model}

The $\mathrm{WAV}_{\mathrm{CNS}}$ index reflects the combined effect of the anesthesia drug and the unknown surgical stimulation. It takes values ranging from 100 to 0 , where $W A V_{C N S}=100$ corresponds to fully awake state, $\mathrm{WAV}_{\mathrm{CNS}}=50$ corresponds to adequate anesthesia, and $\mathrm{WAV}_{\mathrm{CNS}}=0$ corresponds to an isoelectric electroencephalogram (EEG). The $W A V_{C N S}$ monitor model derived by Zikov et al. (Zikov et al., 2006) was used:

$$
y(s)=W A V_{C N S}(s)=100 \times\left\{1-\frac{1}{(8 s+1)^{2}}[E(s)+d(s)]\right\},
$$

where $d$ is the unknown surgical stimulation. Note that maximum $(=1)$ and minimum $(=0)$ clinical effects correspond to $\mathrm{WAV}_{\mathrm{CNS}}=0$ and $\mathrm{WAV}_{\mathrm{CNS}}=100$, respectively.

\subsection{Observer design model}

The observer design model is obtained by locally linearizing the PD model in the neighborhood of the operating regime of $E=0.5$ during the maintenance phase of anesthesia, which yields

$$
E\left(\bar{C}_{e}\right)=\frac{\gamma}{4}\left(\bar{C}_{e}-1\right)+\frac{1}{2}=\frac{\gamma}{4 E C_{50}}\left(C_{e}-E C_{50}\right)+\frac{1}{2} .
$$

Combining (1), (2), (4), and (5) results in the following observer design model, where the state variables $x_{5}$ and $x_{6}$ are associated with the $\mathrm{WAV}_{\mathrm{CNS}}$ monitor model (4):

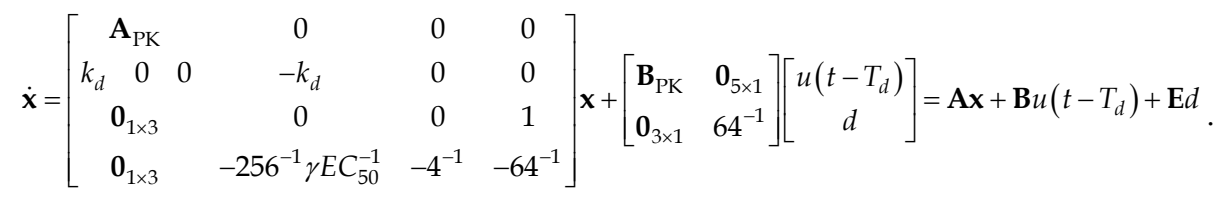

$$
\begin{aligned}
& \mathbf{y}=\left[\begin{array}{llllll}
0 & 0 & 0 & 0 & 1 & 0
\end{array}\right] \mathbf{x}=\mathbf{C} \mathbf{x}
\end{aligned}
$$

\section{Observer design}

A patient undergoing anesthesia is subject to a significant amount of uncertainty arising from the inherent inter-patient physiologic variability and unknown surgical stimulation. The population-based PK and PD models have thus limited predictive accuracy (Schüttler \& Ihmsen, 2000). Besides, the PK and PD model parameters may fluctuate in response to the individual patient's physiologic condition. Furthermore, the clinical effect measurement is persistently corrupted by unpredictable surgical stimulation. The observer must be designed to be robust against these multiple confounding factors.

\subsection{Design strategies}

The following alternatives were considered as observers for drug concentration estimation: 1) RLO: a linear observer with a robust feedback gain, 2) RNO: a nonlinear observer with a robust feedback and a nonlinear disturbance rejection gain, and 3) $\mathrm{H}_{\infty} \mathrm{O}$ : a robust dynamic 
observer designed based on $\mathrm{H}_{\infty}$ control theory. The RLO was intended to explore the limit of performance of a simple linear observer. The RNO was motivated by the effort to separate the tasks of robustness and disturbance rejection: the linear feedback was designed to achieve robustness against PK/PD model uncertainty, whereas the nonlinear feedback was designed to compensate for the unknown surgical stimulation. The requirements of disturbance rejection and the observer matching condition necessitates the use of WAV $\mathrm{CNS}_{\mathrm{C}}$ and its derivative as measurements in the design of RNO. The purpose of the $\mathrm{H}_{\infty} \mathrm{O}$ was to examine if its added complexity is worth exploiting in order to improve the fidelity of drug concentration estimation.

\subsection{Robust linear observer (RLO) design}

A standard linear observer assumes the following form:

$$
\dot{\hat{\mathbf{x}}}=\overline{\mathbf{A}} \hat{\mathbf{x}}+\overline{\mathbf{B}} u+\mathbf{L}(\mathbf{y}-\mathbf{C} \hat{\mathbf{x}}),
$$

where the upper bar on a matrix denotes its nominal value. $\mathbf{L}$ is designed to satisfy the inequality (8) for a positive definite matrix $\mathbf{P}$ in order to place the closed-loop poles in the left-half plane:

$$
(\overline{\mathbf{A}}-\mathbf{L C})^{\mathrm{T}} \mathbf{P}+\mathbf{P}(\overline{\mathbf{A}}-\mathbf{L C})<\mathbf{0} .
$$

Considering the patient model (6) and the observer (7), the propofol concentration estimates provided by the observer are susceptible to the model perturbations included in Ed that stem from the PK/PD uncertainty and unknown surgical stimulation. The observer gain matrix $\mathbf{L}$ must be designed so as to achieve robustness against these confounding factors. It is possible to determine $\mathbf{L}$ such that the state estimation error is bounded within a prescribed ellipsoid in the presence of model perturbations and/or disturbances by exploiting Theorem 1 below:

Theorem 1 (Set-Theoretic Robustness Condition) (Blanchini \& Miani, 2008): Consider the system of the form

$$
\dot{\mathbf{x}}=\mathbf{A x}+\mathbf{F w}
$$

which is subject to a persistent external disturbance $\mathbf{w}$. Assume that the disturbance is bounded by $\mathbf{w}^{\mathrm{T}} \mathbf{W} \leq 1$. Then the ellipsoid $\left\{\mathbf{x}: \mathbf{x}^{\mathrm{T}} \mathbf{P} \mathbf{x} \leq 1\right\}$ is invariant if $\mathbf{Q}=\mathbf{P}^{-1}$ satisfies the condition

$$
\mathbf{Q A}+\mathbf{A}^{\mathrm{T}} \mathbf{Q}+\alpha \mathbf{Q}+\frac{1}{\alpha} \mathbf{F F}^{\mathrm{T}} \leq \mathbf{0}
$$

for some $\alpha>0$.

Rewrite (6) as follows where the matrices $\mathbf{A}$ and $\mathbf{B}$ are decomposed into their nominal and uncertain parts:

$$
\dot{\mathbf{x}}=\mathbf{A} \mathbf{x}+\mathbf{B} u+\mathbf{E} d=(\overline{\mathbf{A}}+\tilde{\mathbf{A}}) \mathbf{x}+(\overline{\mathbf{B}}+\tilde{\mathbf{B}}) u+\mathbf{E} d .
$$

Subtracting (7) from (9) yields the following error dynamics: 


$$
\dot{\mathbf{e}}=(\overline{\mathbf{A}}-\mathbf{L C}) \mathbf{e}+\tilde{\mathbf{A}} \mathbf{x}+\tilde{\mathbf{B}} u+\mathbf{E} d=(\overline{\mathbf{A}}-\mathbf{L C}) \mathbf{e}+\mathbf{F} \mathbf{w} .
$$

where $\mathbf{F}$ specifies the uncertainty bound and is chosen such that $\mathbf{w}^{\mathrm{T}} \mathbf{w} \leq 1$. Then according to Theorem 1 the state estimation error $\mathbf{e}$ is bounded within the ellipsoid $\left\{\mathbf{e}: \mathbf{e}^{\mathrm{T}} \mathbf{P e} \leq 1\right\}$ if $\mathbf{Q}=\mathbf{P}-1$ satisfies the following inequality for some $\alpha>0$ :

$$
\mathbf{Q}(\overline{\mathbf{A}}-\mathbf{L C})^{\mathrm{T}}+(\overline{\mathbf{A}}-\mathbf{L C}) \mathbf{Q}+\alpha \mathbf{Q}+\frac{1}{\alpha} \mathbf{F F}^{\mathrm{T}} \leq \mathbf{0} .
$$

Pre- and post-multiplying (11) by $\mathbf{P}$ results in (12):

$$
(\overline{\mathbf{A}}-\mathbf{L C})^{\mathrm{T}} \mathbf{P}+\mathbf{P}(\overline{\mathbf{A}}-\mathbf{L C})+\alpha \mathbf{P}+\frac{1}{\alpha} \mathbf{P F F}^{\mathrm{T}} \mathbf{P} \leq \mathbf{0} .
$$

Noting that $\mathbf{P}$ and PFFT $\mathbf{P}$ in (12) are positive definite and positive semi-definite, respectively, the inequality (12) is stronger than (8) and the pair of matrices $\mathbf{L}$ and $\mathbf{P}$ satisfying (12) automatically satisfies (8). Also, the "size" of $\mathbf{P}$ is directly related to the size of the error ellipsoid within which the state estimation error is bounded. Therefore, the optimal observer gain $\mathbf{L}$ for (7) can be determined by finding the pair of matrices $\mathbf{L}$ and $\mathbf{P}$ which maximizes the size of $\mathbf{P}$ while satisfying the set-theoretic robustness condition (12).

The optimal RLO gain design problem can be cast into a linear matrix inequality (LMI) problem by manipulating (12) as follows. The inequality (12) implies that, for any $\mathbf{e} \neq 0$,

$$
\mathbf{e}^{\mathrm{T}}\left[\left(\overline{\mathbf{A}}+\frac{1}{2} \alpha \mathbf{I}-\mathbf{L C}\right)^{\mathrm{T}} \mathbf{P}+\mathbf{P}\left(\overline{\mathbf{A}}+\frac{1}{2} \alpha \mathbf{I}-\mathbf{L C}\right)\right] \mathbf{e}+\frac{1}{\alpha} \mathbf{e}^{\mathrm{T}} \mathbf{P F} \mathbf{F}^{\mathrm{T}} \mathbf{P e} \leq \mathbf{0} .
$$

Define $\mathbf{Y}=\mathbf{P L}$. Using

$$
-\alpha \mathbf{w}^{\mathrm{T}} \mathbf{w}+2 \mathbf{e}^{\mathrm{T}} \mathbf{P F} \mathbf{w}=-\alpha \mathbf{w}^{\mathrm{T}} \mathbf{w}+2 \mathbf{e}^{\mathrm{T}} \mathbf{P F} \mathbf{w}-\frac{1}{\alpha} \mathbf{e}^{\mathrm{T}} \mathbf{P F F} \mathbf{T}^{\mathrm{T}} \mathbf{P e}+\frac{1}{\alpha} \mathbf{e}^{\mathrm{T}} \mathbf{P F F}{ }^{\mathrm{T}} \mathbf{P e} \leq \frac{1}{\alpha} \mathbf{e}^{\mathrm{T}} \mathbf{P F F}^{\mathrm{T}} \mathbf{P e},
$$

the inequality (13) can be converted to the following LMI:

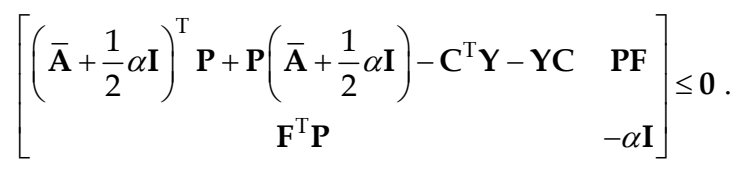

Summarizing, the optimal RLO gain design is equivalent to solving the following LMI:

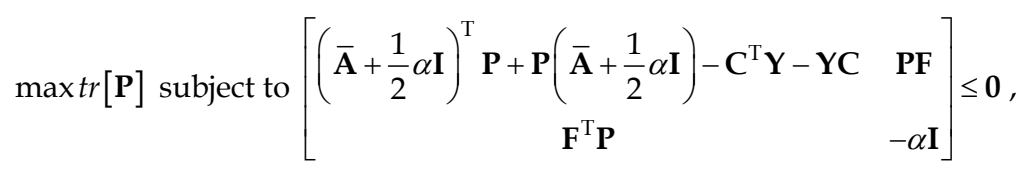

where the size of $\mathbf{P}$ is measured in terms of its trace norm, which is equal to the sum of its eigenvalues. 


\subsection{Robust nonlinear observer (RNO) design}

The RNO to be designed in this chapter assumes the following form:

$$
\dot{\hat{\mathbf{x}}}=\overline{\mathbf{A}} \hat{\mathbf{x}}+\overline{\mathbf{B}} u+\mathbf{L}(\mathbf{y}-\mathbf{C} \hat{\mathbf{x}})+\mathbf{\eta},
$$

where $\mathbf{L}$ is the linear feedback designed to suppress the adverse effect of the PK/PD uncertainty, and $\eta$ is the nonlinear feedback to compensate for the unknown surgical stimulation. The error dynamics is derived by subtracting (17) from (9):

$$
\dot{\mathbf{e}}=(\overline{\mathbf{A}}-\mathbf{L C}) \mathbf{e}+\tilde{\mathbf{A}} \mathbf{x}+\tilde{\mathbf{B}} u+\mathbf{E} d=(\overline{\mathbf{A}}-\mathbf{L C}) \mathbf{e}+\mathbf{G} \mathbf{w}+\mathbf{E} d-\mathbf{\eta},
$$

where $\mathbf{G}$ specifies the uncertainty bound and is chosen such that $\mathbf{w}^{\mathrm{T}} \mathbf{w} \leq 1$. Note that

$$
\mathbf{F} \mathbf{w}=\mathbf{G} \mathbf{w}+\mathbf{E} d,
$$

which suggests that within the RNO framework the PK/PD uncertainty $\mathrm{Gw}$ is taken care of by $\mathbf{L}$, whereas the surgical stimulation is accounted for by $\eta$, in contrast to the RLO where both of these confounding factors $\mathbf{F w}=\mathrm{Gw}+\mathrm{Ed}$ must be dealt with by $\mathbf{L}$.

To exploit $\eta$ to cancel out the effect of $d$, it is required that the following observer matching condition be satisfied for some function $\mathbf{h}(\mathbf{x}, \mathrm{u})$ :

$$
\mathbf{E} d=\mathbf{P}^{-1} \mathbf{C}^{\mathrm{T}} \mathbf{h}(\mathbf{x}, u)
$$

where $\mathbf{P}$ satisfies (8). Note that (20) cannot be satisfied by the output matrix $\mathbf{C}$ in (6); it violates the positive definite requirement on $\mathbf{P}$ by imposing $\mathrm{p}_{66}=0$. In order to resolve this problem, we assume that $\mathrm{WAV}_{\mathrm{CNS}}$ and its time derivative are available as sensor measurements:

$$
\mathbf{y}=\left[\begin{array}{ll}
\mathbf{0}_{4 \times 4} & \mathbf{I}_{2 \times 2}
\end{array}\right] \mathbf{x}=\mathbf{D x} .
$$

With this choice of the output, the following constraints are imposed on $\mathbf{P}$ :

$$
p_{16}=p_{26}=p_{36}=p_{46}=0, p_{56} d=64 h_{1}(\mathbf{x}, u), p_{66} d=64 h_{2}(\mathbf{x}, u) .
$$

Then the linear feedback gain $\mathbf{L}$ of the RNO can be designed by solving the LMI (16) with $\mathbf{F}$ substituted by $\mathbf{G}$, subject to (22). Once a feasible $\mathbf{L}$ and $\mathbf{P}$ are obtained from the LMI, the function $\mathbf{h}(\mathbf{x}, \mathbf{u})$ is directly obtained from (22).

Assuming that the linear feedback gain $\mathbf{L}$ and the positive matrix $\mathbf{P}$ are designed to satisfy (16) with $\mathbf{F}$ substituted by $\mathbf{G}$ and the observer matching condition (20), the nonlinear feedback $\eta$ with $\rho$ satisfying $\rho>|| \mathbf{h}(\mathbf{x}, \mathrm{u}) \|$ guarantees the boundedness of the observer error $\mathbf{e}$ :

$$
\mathbf{\eta}=\rho \frac{\mathbf{P}^{-1} \mathbf{D}^{\mathrm{T}} \mathbf{D e}}{\|\mathbf{D e}\|},
$$

which can be shown by considerng the Lyapunov function $\mathrm{V}(\mathbf{e})=\mathbf{e}^{\mathrm{T}} \mathbf{P e}$. Using the error dynamics (18), the time derivative of $\mathrm{V}(\mathbf{e})$ becomes: 


$$
\begin{aligned}
\dot{V}(\mathbf{e}) & =\dot{\mathbf{e}}^{\mathrm{T}} \mathbf{P e}+\mathbf{e}^{\mathrm{T}} \mathbf{P e} \\
& =\mathbf{e}^{\mathrm{T}}\left[(\overline{\mathbf{A}}-\mathbf{L D})^{\mathrm{T}} \mathbf{P}+\mathbf{P}(\overline{\mathbf{A}}-\mathbf{L D})\right] \mathbf{e}+2 \mathbf{e}^{\mathrm{T}} \mathbf{P G} \mathbf{w}+2 \mathbf{e}^{\mathrm{T}} \mathbf{P E} d-2 \mathbf{e}^{\mathrm{T}} \mathbf{P} \mathbf{\eta} \\
& \leq-\alpha \mathbf{e}^{\mathrm{T}} \mathbf{P e}-\frac{1}{\alpha} \mathbf{e}^{\mathrm{T}} \mathbf{P G} \mathbf{G}^{\mathrm{T}} \mathbf{P e}+2 \mathbf{e}^{\mathrm{T}} \mathbf{P G} \mathbf{w}+2 \mathbf{e}^{\mathrm{T}} \mathbf{P E} d-2 \mathbf{e}^{\mathrm{T}} \mathbf{P} \mathbf{\eta} \\
& \leq \underbrace{-\alpha\left(\mathbf{e}^{\mathrm{T}} \mathbf{P e}-\mathbf{w}^{\mathrm{T}} \mathbf{w}\right)}_{(\mathrm{a})}+\underbrace{2\|\mathbf{D e}\|(\|\mathbf{h}(\mathbf{x}, u)\|-\rho)}_{(\mathrm{b})}
\end{aligned}
$$

Note that 1 ) the term (a) is strictly negative whenever $\mathbf{e}$ is outside of the ellipsoid defined by $\left\{\mathbf{e}: \mathbf{e}^{\mathrm{T}} \mathbf{P e} \leq 1\right\}$, and 2) the term (b) is always strictly negative. Thus, the time derivative of $\mathrm{V}(\mathbf{e})$ is strictly negative if $\mathbf{e}^{\mathrm{T}} \mathbf{P e} \leq 1$ is violated. Therefore, the observer error is guaranteed to converge to and be bounded within the ellipsoid $\left\{\mathbf{e}: \mathbf{e}^{\mathrm{T}} \mathbf{P e} \leq 1\right\}$.

\section{4 $\mathrm{H}_{\infty}$ observer $\left(\mathrm{H}_{\infty} \mathrm{O}\right)$ design}

The $\mathrm{H}_{\infty} \mathrm{O}$ was designed as described in Hahn et al. (2011) and the detailed discussion on its design is therefore omitted here. Briefly, the $\mathrm{H}_{\infty} \mathrm{O}$ assumes the same form as the RLO (6). However, in contrast to the RLO whose observer gain $\mathbf{L}$ is a constant matrix, the observer gain $\mathbf{L}$ of the $\mathrm{H}_{\infty} \mathrm{O}$ is a transfer matrix: $\mathbf{L}=\mathbf{L}(\mathrm{s})$, where $\mathrm{s}$ is the Laplace variable. Therefore, the state estimation is given by:

$$
\dot{\hat{\mathbf{x}}}=\overline{\mathbf{A}} \hat{\mathbf{x}}+\overline{\mathbf{B}} u+\mathbf{v},
$$

where the feedback $v$ is the output of a dynamic system with the clinical effect estimation error as its excitation signal:

$$
\dot{\boldsymbol{\xi}}=\mathbf{A}_{\xi} \boldsymbol{\xi}+\mathbf{B}_{\xi}(y-\mathbf{C} \hat{\mathbf{x}}), \mathbf{v}=\mathbf{C}_{\xi} \boldsymbol{\xi}+\mathbf{D}_{\xi}(y-\mathbf{C} \hat{\mathbf{x}}) .
$$

The transfer matrix between $v$ and $\tilde{y}=y-\mathbf{C} \hat{\mathbf{x}}$ becomes

$$
\mathbf{L}(s)=\mathbf{C}_{\xi}\left[s \mathbf{I}-\mathbf{A}_{\xi}\right]^{-1} \mathbf{B}_{\xi}+\mathbf{D}_{\xi} .
$$

The objective is to design a feedback gain $\mathbf{L}(\mathrm{s})$ of the $\mathrm{H}_{\infty} \mathrm{O}$ that is robust against Fw. In the frequency domain, (6) and (25) are expressed as follows:

$$
\begin{aligned}
& \mathbf{x}(s)=[s \mathbf{I}-\overline{\mathbf{A}}]^{-1} \overline{\mathbf{B}} u(s)+[s \mathbf{I}-\overline{\mathbf{A}}]^{-1} \mathbf{F w}(s) \\
& \hat{\mathbf{x}}(s)=[s \mathbf{I}-\overline{\mathbf{A}}]^{-1} \overline{\mathbf{B}} u(s)+[s \mathbf{I}-\overline{\mathbf{A}}]^{-1} \mathbf{L}(s) \tilde{y}(s)
\end{aligned} .
$$

where $\tilde{y}(s)=y(s)-\mathbf{C} \hat{\mathbf{x}}(s)$. Subtracting $\hat{\mathbf{x}}(s)$ from $\mathbf{x}(\mathrm{s})$ yields the following error dynamics:

$$
\mathbf{e}(s)=\mathbf{T}_{\mathbf{e w}}(s) \mathbf{w}(s)=\left\{\mathbf{I}+[s \mathbf{I}-\overline{\mathbf{A}}]^{-1} \mathbf{L}(s) \mathbf{C}\right\}^{-1}[s \mathbf{I}-\overline{\mathbf{A}}]^{-1} \mathbf{F w}(s) .
$$

Based on (29), the $\mathrm{H}_{\infty} \mathrm{O}$ can be designed by minimizing the $\mathrm{H}_{\infty}$ norm of the frequencyweighted closed-loop disturbance-to-error transfer function $\mathbf{T}_{\mathbf{e w}}(\mathrm{s})$ : 


$$
\min _{\mathbf{L}(j \omega)}\left\|\mathbf{W}_{\mathbf{e}}(j \omega) \mathbf{T}_{\mathbf{e w}}(j \omega)\right\|_{\infty}
$$

where $\mathbf{W}_{\mathbf{e}}(\mathrm{s})$ is a weighting function specifying the desired error bound. The linear fractional transformation (LFT) (Skogestad \& Postlethwaite, 1996) can be used to convert (30) to the standard $\mathrm{H}_{\infty}$ optimization set-up (Skogestad \& Postlethwaite, 1996) shown in Fig. 4, where $\mathbf{P}(\mathrm{s})$ is given by:

$$
\left[\begin{array}{c}
\mathbf{z}(s) \\
\tilde{y}(s)
\end{array}\right]=\mathbf{P}(s)\left[\begin{array}{c}
\mathbf{w}(s) \\
\mathbf{v}(s)
\end{array}\right]=\left[\begin{array}{cc}
\mathbf{W}_{\mathbf{e}}(s)[s \mathbf{I}-\overline{\mathbf{A}}]^{-1} \mathbf{F} & -\mathbf{W}_{\mathbf{e}}(s)[s \mathbf{I}-\overline{\mathbf{A}}]^{-1} \\
\mathbf{C}[s \mathbf{I}-\overline{\mathbf{A}}]^{-1} \mathbf{F} & -\mathbf{C}[s \mathbf{I}-\overline{\mathbf{A}}]^{-1}
\end{array}\right]\left[\begin{array}{c}
\mathbf{w}(s) \\
\mathbf{v}(s)
\end{array}\right],
$$

where $\mathbf{z}(\mathrm{s})=\mathbf{W}_{\mathbf{e}}(\mathrm{s}) \mathbf{e}(\mathrm{s})$. This chapter employed a simple first-order filter (32) as $\mathbf{W}_{\mathbf{e}}(\mathrm{s})$, which aims at small errors in the low frequency region:

$$
\mathbf{W}_{\mathbf{e} 4}(s)=k \frac{\tau_{2} s+1}{\tau_{1} s+1} \times \frac{1}{2 E C_{50}} \times\left[\begin{array}{ll}
\mathbf{I}_{4 \times 4} & \mathbf{0}_{4 \times 2}
\end{array}\right],
$$

where $\mathrm{k}$ specifies the error bound at steady state, $\tau_{1}$ specifies the observer bandwidth, and $\tau_{2}>\tau_{1}$. Then the state-space representation (33) of $\mathbf{P}(\mathrm{s})$ is obtained:

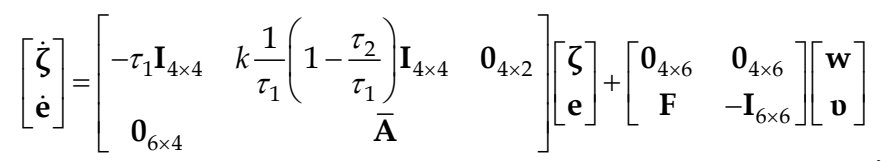

$$
\begin{aligned}
& {\left[\begin{array}{l}
\mathbf{z} \\
\tilde{y}
\end{array}\right]=\left[\begin{array}{lrr}
\mathbf{I}_{4 \times 4} & k \frac{\tau_{2}}{\tau_{1}} \mathbf{I}_{4 \times 4} & \mathbf{0}_{4 \times 2} \\
\mathbf{0}_{1 \times 4} & & \mathbf{C}
\end{array}\right]\left[\begin{array}{l}
\boldsymbol{\zeta} \\
\mathbf{e}
\end{array}\right]+\left[\begin{array}{ll}
\mathbf{0}_{4 \times 6} & \mathbf{0}_{4 \times 6} \\
\mathbf{0}_{1 \times 6} & \mathbf{0}_{1 \times 6}
\end{array}\right]\left[\begin{array}{l}
\mathbf{w} \\
\mathbf{v}
\end{array}\right]}
\end{aligned}
$$

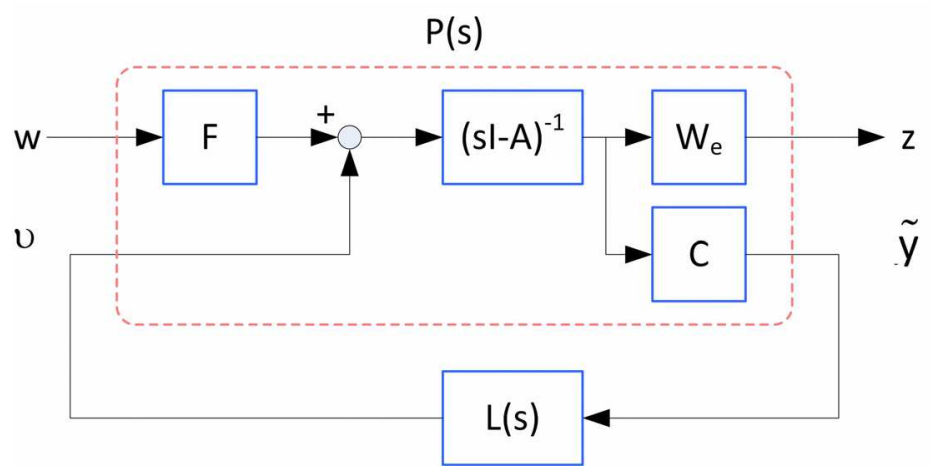

Fig. 4. Standard $\mathrm{H}_{\infty}$ optimization set-up for $\mathrm{H}_{\infty} \mathrm{O}$ design.

Based on (33), the optimal $\mathrm{H}_{\infty} \mathrm{O}$ can be designed using the D-K iteration (Skogestad \& Postlethwaite, 1996). Note that although (33) does not satisfy all the regularity assumptions required for $\mathrm{H}_{\infty}$ optimization (Skogestad \& Postlethwaite, 1996), the problem can be resolved by any standard regularization procedure (e.g. Pertew et al., 2006). 


\section{Methods}

\subsection{Design of observers}

The demographic and PD data of 15 patients aged 18-30 (22+/-3.2) listed in Dumont et al. (2009) were used to obtain a nominal PK and PD model (1)-(4). Then 100 random MonteCarlo models were created for the nominal model by applying up to $+/-15 \%$ perturbations to all the PK and PD parameters. The PK/PD variability was specified as follows:

$$
\mathbf{F} \mathbf{w}=\tilde{\mathbf{A}} \mathbf{x}+\tilde{\mathbf{B}} u+\mathbf{E} d=\operatorname{diag}\left\{\delta_{i}\right\} \mathbf{w}
$$

where $\delta_{i}, i=1, \ldots, 6$ were chosen to encompass the resultant effect of up to $+/-15 \%$ random $\mathrm{PK} / \mathrm{PD}$ parametric perturbations as well as surgical stimulation of up to $+/-20 \mathrm{WAV}_{\mathrm{CNS}}$ units.

Before designing the observers, the observability of the system was analyzed by evaluating the observability grammian matrix (35) with the nominal patient model:

$$
\mathbf{W}_{o}=\int_{0}^{\infty} \exp \left[\mathbf{A}^{\mathrm{T}} \tau\right] \mathbf{C}^{\mathrm{T}} \mathbf{C} \exp [\mathbf{A} \tau] d \tau,
$$

using which the relationship between the dominant direction of each eigenvalue and the propofol concentration at each compartment was examined. The condition number of $\mathbf{W}_{\mathrm{o}}$ was also calculated to assess the well-posedness of the observer design problem.

Using the nominal patient model, the observer gain matrix $\mathbf{L}$ for RLO and RNO were designed by solving the LMI (8) and (16) with $\alpha=1$ and $\alpha=10^{-5}$, respectively. In the RNO design, the constraint (22) was imposed on $\mathbf{P}$. The $\mathrm{H}_{\infty} \mathrm{O}$ was designed by applying the D-K iteration procedure to the standard set-up (33). The weighting function (32) was specified as $\mathrm{k}=5.0, \tau_{1}^{-1}=2.0 \times 10^{-5} \mathrm{~Hz}$, and $\tau_{2}^{-1}=1.0 \times 10^{9} \mathrm{~Hz}$, which requires the error to be bounded by $20 \%$ up to $2.0 \times 10^{-5} \mathrm{~Hz}$, beyond which an increase in size of the error is permitted.

\subsection{Monte-Carlo simulation}

The 100 randomly created patient models were used for the Monte-Carlo simulation. TCI administration of propofol to target the effect site propofol concentration of $3 \mathrm{mcg} / \mathrm{ml}$ was chosen as the simulation scenario (Fig. 5). The duration of the simulated procedure was 2000s. The surgical stimulation shown in Dumont et al. (2009) was adopted and applied to the patient models from 1200s to 1700s (Fig. 5). The open-loop-predicted propofol concentrations were calculated by solving the PK and PD models (1) and (2).

\subsection{Statistical analysis}

From the simulation of each of the 100 random patient models, the following plasma and effect site percentage errors (PE) were calculated for both observer-based estimation (RLO, $\mathrm{RNO}$, and $\mathrm{H}_{\infty} \mathrm{O}$ ) and open-loop prediction:

$$
P E_{j}^{s}=100 \times \frac{C_{s}(j)-\hat{C}_{s}(j)}{C_{s}(j)},
$$


where $P_{\mathrm{j}} \mathrm{s}$ denotes the PE for the sample $\mathrm{j}$ at the site $\mathrm{s}(\mathrm{s}=\mathrm{p}$ for plasma and $\mathrm{s}=\mathrm{e}$ for effect site), and $C_{s}(j)$ and $\hat{C}_{s}(j)$ are the true versus open-loop predicted or closed-loop estimated drug concentrations for the sample $j$ at the site s. The median absolute PE (MDAPE), mean absolute PE (MNAPE) and maximum absolute PE (MXAPE) were calculated as follows and were used to compare the performance of the observers and its potential over the open-loop prediction: $\mathrm{MDAPE}_{\mathrm{s}}=\operatorname{median}\left\{\left|\mathrm{PE}_{\mathrm{j}} \mathrm{s}\right|\right\}, \mathrm{MNAPE}_{\mathrm{s}}=\operatorname{mean}\left\{\left|P \mathrm{PE}_{\mathrm{j}} \mathrm{s}\right|\right\}$, and $\mathrm{MXAPE}_{\mathrm{s}}=\max \left\{\left|P E_{j} \mathrm{~s}\right|\right\}$ (Varvel et al., 1992). The errors were calculated for the induction phase of anesthesia $(t<600 s)$ and the entire procedure $(t<2000 s)$ in order to examine the effectiveness of the observers against surgical stimulation. The reduction in errors by the observers over the open-loop prediction was assessed in terms of the median value of the 100 observerestimated MDAPE, MNAPE and MXAPE normalized by their open-loop-predicted counterparts. The statistical significance of the error reduction was determined by applying the two-sample t-test to the 100 pairs of observer-estimated and open-loop predicted MDAPE, MNAPE and MXAPE.
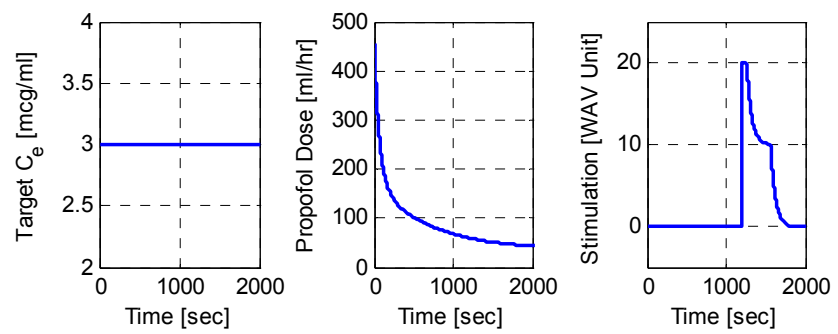

Fig. 5. Propofol effect site TCI scenario for Monte-Carlo simulation.

\section{Results and discussion}

\subsection{Observability analysis and observer design}

The effect site propofol concentration $x_{4}$ (which is the primary concentration of interest) was associated with the second smallest eigenvalue of $\mathbf{W}_{0}$, whereas the peripheral compartmental concentrations $\mathrm{x}_{2}$ and $\mathrm{x}_{3}$ corresponded to its largest and second largest eigenvalues. This implies that the influence of $x_{4}$ on the output $\left(x_{5}\right)$ is relatively weak compared to $x_{2}$ and $x_{3}$, because $x_{2}$ and $x_{3}$ can dominate $x_{4}$ in terms of output energy through their slowly decaying dynamics (i.e. $x_{2}$ and $x_{3}$ decay much slower than $x_{1}$ and $x_{4}$ ). The condition number of $\mathbf{W}_{\mathrm{o}}$ was very large (approximately 821 ), indicating that the degree of observability for propofol concentrations in different compartments can be significantly different. Essentially, the observability analysis clearly illustrates that estimating $x_{4}$ is inherently a challenging task.

RLO design resulted in $\mathbf{L}$ with optimal $\operatorname{tr}[\mathbf{P}]=6.7 \times 10^{8}$. The real part of the closed-loop eigenvalues ranged from -4.5 to -0.4 . RNO design resulted in $\mathbf{L}$ with optimal $\operatorname{tr}[\mathbf{P}]=8.2 \times 10^{8}$. The real parts of the closed-loop eigenvalues range from $-2.1 \times 10^{2}$ to $-1.2 \times 10^{-4} . \mathrm{H}_{\infty} \mathrm{O}$ design resulted in $\mathbf{L}(\mathrm{s})$ that has robust stability margin of $\infty$ and robust performance margin of 1.1, indicating that the closed-loop system is always stable, and is robust against up to 1.1 times the uncertainty incorporated in the observer design (Balas et al., 2009). 


\subsection{Performance evaluation and statistical analysis}

The distributions of MDAPE, MNAPE, and MXAPE of the propofol concentration errors at the plasma and the effect site are shown in Fig. 6 and Fig. 7 for the induction phase of anesthesia and the entire procedure, respectively. Tables 1-3 summarize mean (SD) of these errors.
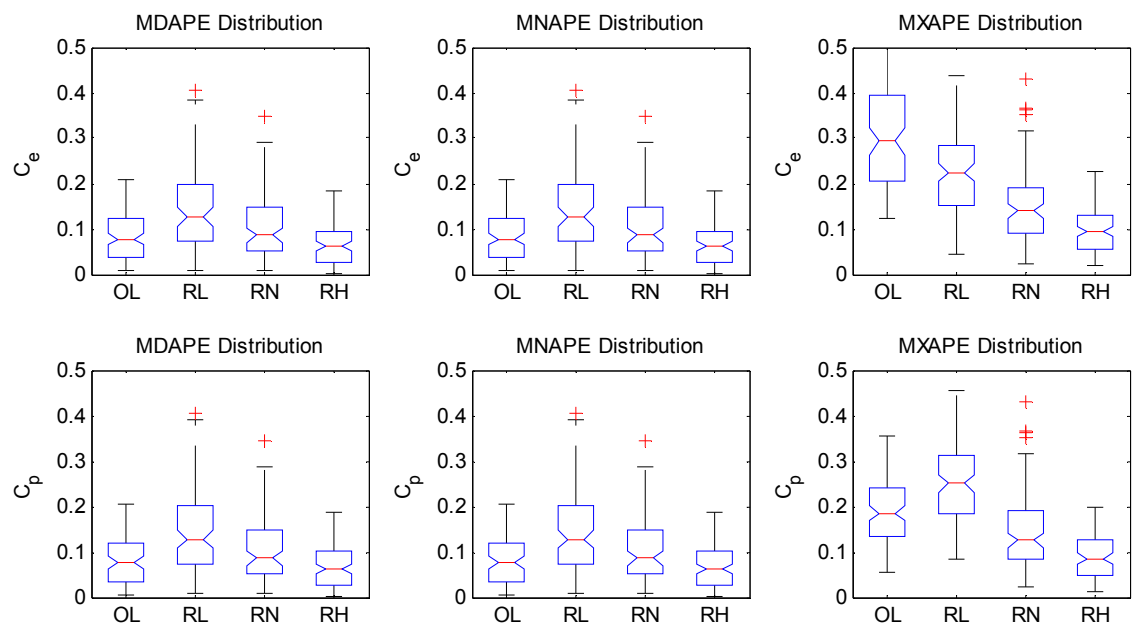

Fig. 6. Distributions of MDAPE, MNAPE, MXAPE during the induction phase of anesthesia. OL: open-loop prediction, RL: RLO, RN: RNO, RH: $\mathrm{H}_{\infty} \mathrm{O}$.
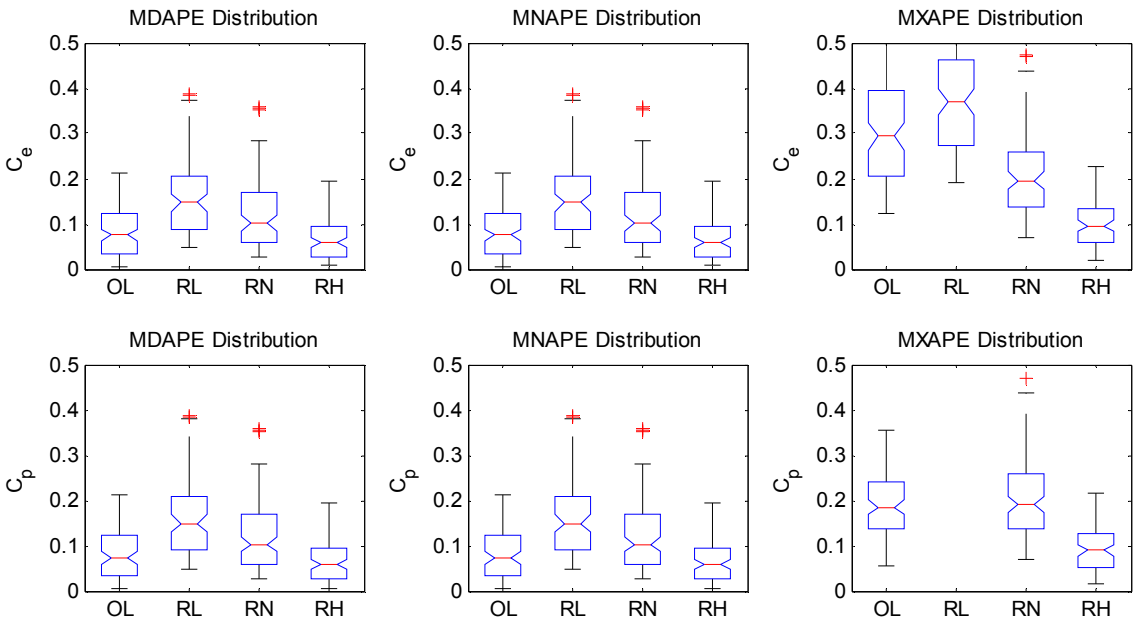

Fig. 7. Distributions of MDAPE, MNAPE, MXAPE during the entire procedure. OL: openloop prediction, RL: RLO, RN: $\mathrm{RNO}, \mathrm{RH}: \mathrm{H}_{\infty} \mathrm{O}$. 
The RNO and the $\mathrm{H}_{\infty} \mathrm{O}$ outperformed the RLO. Except for its benefit in reducing MXAPE of $\mathrm{C}_{\mathrm{e}}$ during the induction phase of anesthesia (by 21\%, p<10-5; Table 3), the RLO was no better than the open-loop prediction (Table 1 and Table 2). Compared with the open-loop prediction, the RNO was capable of reducing MXAPE of $\mathrm{C}_{\mathrm{e}}$ (by $59 \%, \mathrm{p}<10^{-5}$ ) and $\mathrm{C}_{\mathrm{p}}$ (by $38 \%$, $\mathrm{p}<10^{-5}$ ) during the induction phase of anesthesia (Table 3), and MXAPE of $\mathrm{C}_{\mathrm{e}}$ (by $43 \%, \mathrm{p}<10^{-}$ 5) during the entire procedure (Table 3). Its MDAPE and MNAPE were as good as those of the open-loop prediction during the induction phase of anesthesia, but they deteriorated more than the open-loop prediction when evaluated for the entire procedure (Table 1 and Table 2). The degraded performance of the observers in the presence of surgical stimulation is expected because it distorts the clinical effect measurement, resulting in an inappropriate feedback correction, whereas the open-loop prediction is not affected by surgical stimulation (see Fig. 1). In contrast to $\mathrm{RLO}$ and $\mathrm{RNO}$, the $\mathrm{H}_{\infty} \mathrm{O}$ was able to deliver statistically significant reduction in all the PE metrics over the open-loop prediction, regardless of the presence of surgical stimulation (see Table 4). Moreover, the SD of all the metrics for the $\mathrm{H}_{\infty} \mathrm{O}$ were significantly smaller than those of the open-loop prediction, indicating that the propofol concentrations provided by the $\mathrm{H}_{\infty} \mathrm{O}$ are more reliable. This suggests that, in contrast to the open-loop prediction in which the PK/PD variability directly deteriorates the propofol concentration estimates, the observer's feedback correction based on the clinical effect measurement is viable in suppressing the propagation of the adverse influences of the $\mathrm{PK} / \mathrm{PD}$ variability into the propofol concentration errors.

\begin{tabular}{c||c|c|c|c||c|c|c|c}
\hline \hline \multicolumn{1}{c||}{} & \multicolumn{4}{c||}{ Induction Phase } & \multicolumn{4}{c}{ Entire Procedure } \\
\cline { 2 - 9 } & OL & RLO & RNO & $\mathrm{H}_{\infty} \mathrm{O}$ & OL & RLO & RNO & $\mathrm{H}_{\infty} \mathrm{O}$ \\
\hline \hline $\mathrm{C}_{\mathrm{e}}$ & $8.08(5.61)$ & $14.6(9.91)$ & $9.70(6.81)$ & $7.07(4.96)$ & $8.67(6.06)$ & $15.1(10.2)$ & $11.6(7.78)$ & $6.82(4.99)$ \\
$\mathrm{C}_{\mathrm{p}}$ & $8.07(5.65)$ & $14.6(9.95)$ & $9.70(6.83)$ & $7.18(5.06)$ & $8.66(6.07)$ & $15.1(10.1)$ & $11.6(7.77)$ & $6.93(5.07)$ \\
\hline \hline
\end{tabular}

Table 1. Distribution of MDAPE: mean (SD).

\begin{tabular}{c||c|c|c|c||c|c|c|c}
\hline \hline \multicolumn{1}{c||}{} & \multicolumn{4}{c||}{ Induction Phase } & \multicolumn{4}{c}{ Entire Procedure } \\
\cline { 2 - 9 } & OL & RLO & RNO & $\mathrm{H}_{\infty} \mathrm{O}$ & OL & RLO & RNO & $\mathrm{H}_{\infty} \mathrm{O}$ \\
\hline \hline $\mathrm{C}_{\mathrm{e}}$ & $8.70(5.06)$ & $14.8(9.47)$ & $9.51(6.44)$ & $7.01(4.80)$ & $8.68(5.71)$ & $16.3(8.94)$ & $12.3(7.60)$ & $6.78(4.84)$ \\
$\mathrm{C}_{\mathrm{p}}$ & $8.24(5.18)$ & $14.9(9.58)$ & $9.48(6.42)$ & $7.11(4.90)$ & $8.55(5.76)$ & $16.6(9.02)$ & $12.3(7.58)$ & $6.89(4.94)$ \\
\hline \hline
\end{tabular}

Table 2. Distribution of MNAPE: mean (SD).

\begin{tabular}{c||c|c|c|c||c|c|c|c}
\hline \hline \multicolumn{1}{c||}{} & \multicolumn{4}{c||}{ Induction Phase } & \multicolumn{4}{c}{ Entire Procedure } \\
\cline { 2 - 8 } & OL & RLO & RNO & $\mathrm{H}_{\infty} \mathrm{O}$ & OL & RLO & RNO & $\mathrm{H}_{\infty} \mathrm{O}$ \\
\hline \hline $\mathrm{C}_{\mathrm{e}}$ & $29.7(10.8)$ & $22.1(9.40)$ & $13.9(7.29)$ & $9.44(4.81)$ & $29.8(10.7)$ & $38.1(12.1)$ & $20.1(8.61)$ & $10.1(4.94)$ \\
$\mathrm{C}_{\mathrm{p}}$ & $18.9(7.11)$ & $25.1(9.09)$ & $13.2(7.28)$ & $8.85(4.83)$ & $19.2(6.98)$ & $582 .(53.7)$ & $19.9(8.70)$ & $9.61(4.93)$ \\
\hline \hline
\end{tabular}

Table 3. Distribution of MXAPE: mean (SD). 


\begin{tabular}{c||ccc||ccc}
\hline \hline \multicolumn{1}{c||}{} & \multicolumn{3}{c||}{ Induction Phase } & \multicolumn{3}{c}{ Entire Procedure } \\
\cline { 2 - 7 } & MDAPE & MNAPE & MXAPE & MDAPE & MNAPE & MXAPE \\
\hline \hline $\mathrm{C}_{\mathrm{e}}$ & $13 \%\left(\mathrm{p}<10^{-10}\right)$ & $21 \%\left(\mathrm{p}<10^{-10}\right)$ & $73 \%\left(\mathrm{p}<10^{-10}\right)$ & $24 \%\left(\mathrm{p}<10^{-10}\right)$ & $25 \%\left(\mathrm{p}<10^{-10}\right)$ & $72 \%\left(\mathrm{p}<10^{-10}\right)$ \\
$\mathrm{C}_{\mathrm{p}}$ & $11 \%\left(\mathrm{p}<10^{-10}\right)$ & $15 \%\left(\mathrm{p}<10^{-10}\right)$ & $59 \%\left(\mathrm{p}<10^{-10}\right)$ & $22 \%\left(\mathrm{p}<10^{-10}\right)$ & $21 \%\left(\mathrm{p}<10^{-10}\right)$ & $56 \%\left(\mathrm{p}<10^{-10}\right)$ \\
\hline \hline
\end{tabular}

Table 4. Reduction of PE metrics over open-loop prediction by $\mathrm{H}_{\infty} \mathrm{O}$.

Several important observations can be made from the overall results. First, the observers were effective in reducing MXAPE (except for the RLO in the presence of surgical stimulation, i.e. during the maintenance phase). This is attributed to the philosophy behind the design of robust observers - the observers considered in this chapter were designed to improve the worst-case performance (Skogestad \& Postlethwaite, 1996). Due to the design objective of guaranteeing performance and robustness even in extreme situations, the behaviour of these observers on patients whose dynamics are rather close to the nominal design model may not be optimal (this may partly explain why MDAPE and MNAPE metrics of RLO and RNO are not as good as the open-loop prediction).

Second, in spite of its robustified feedback gain, the standard linear observer was not an attractive strategy for anesthesia drug concentration estimation, due to its limited performance in the presence of surgical stimulation. In our attempt to overcome this limitation, we presented an RNO and an $\mathrm{H}_{\infty} \mathrm{O}$ as alternatives to the RLO. The RNO could provide a marginal improvement that was not sufficient to justify the cost of additional measurement (i.e. the time derivative of the clinical effect). The $\mathrm{H}_{\infty} \mathrm{O}$ exhibited significantly superior performance to RLO and RNO without any extra measurement requirements, by virtue of the additional flexibility in its structure: the feedback gain $\mathrm{L}(\mathrm{s})$ is a dynamic system rather than a constant matrix as in RLO and RNO. Despite their limited performance in the presence of surgical stimulation, RLO and RNO can still be considered as valid options for applications in the induction phase of anesthesia and the operations involving minor surgical procedures, by virtue of their structural simplicity in implementation in comparison with $\mathrm{H}_{\infty} \mathrm{O}$.

From the design perspective, one advantage of the observer-based strategies investigated in this chapter is the explicit specification of the amount of PK/PD uncertainty and surgical stimulation it must tolerate (see the matrix $\mathbf{F}$ in (16) for RLO and $\mathrm{RNO}$ and in (33) for $\mathrm{H}_{\infty} \mathrm{O}$ ) and its systematic use for optimizing the observer performance so that the observer is tuned to appropriately exploit the clinical effect measurement distorted by surgical stimulation, in order to better estimate the drug concentrations over the open-loop prediction in spite of the PK/PD uncertainty.

\section{Conclusion}

This chapter investigated alternative observer-based strategies for estimation of anesthesia drug concentrations. The design objective was dedicated to robustness of observers against the PK/PD model uncertainty and the unknown surgical stimulation. In this chapter, we considered linear (RLO), nonlinear ( $\mathrm{RNO})$, and dynamic $\left(\mathrm{H}_{\infty} \mathrm{O}\right)$ observers. The performance of the observers in comparison with traditional open-loop prediction was evaluated using a 
Monte-Carlo simulation of a surgical procedure. Based on the PE-based metrics, it was concluded that the use of $\mathrm{H}_{\infty} \mathrm{O}$ is optimal. However, RLO and RNO can be viable alternatives for the induction phase of anesthesia and/or minimally stimulating procedures.

\section{Acknowledgment}

This research was supported in part by Natural Sciences and Engineering Research Council of Canada and Canadian Institute of Health Research under their Collaborative Health Research Project program.

\section{References}

Balas, G., Chiang, R., Packard, A., \& Safonov, M. (2009). Robust Control Toolbox User's Guide, MathWorks, Natick, U.S.A.

Blanchini, F., \& Miani, S. (2008). Set-Theoretic Methods in Control, Birkhäuser, ISBN 978-08176-3255-7, Boston, U.S.A.

Dumont, G.A., Martinez, A., \& Ansermino, J.M. (2009). Robust Control of Depth of Anesthesia. International Journal of Adaptive Control and Signal Processing, Vol. 23, No. 5, pp. 435-454, ISSN 0890-6327.

Gentilini, A., Schaniel, C., Morari, M., Bieniok, C., Wymann, R., \& Schnider, T. (2002). A New Paradigm for the Closed-Loop Intraoperative Administration of Analgesics in Humans. IEEE Transactions on Biomedical Engineering, Vol. 49, No. 4, pp. 289-299, ISSN 0018-9294.

Hahn, J.O., Dumont, G.A., \& Ansermino, J.M. (2011). Closed-Loop Anesthetic Drug Concentration Estimation Using Clinical Effect Feedback. IEEE Transactions on Biomedical Engineering, Vol. 58, No. 1, pp. 3-6, ISSN 0018-9294.

Pertew, A.M., Marquez, H.J., \& Zhao, Q. (2006). $\mathrm{H}_{\infty}$ Observer Design for Lipschitz Nonlinear Systems. IEEE Transactions on Automatic Control, Vol. 51, No. 7, pp. 1211-1216, ISSN 0018-9286.

Rao, R.R., Bequette, B.W., \& Roy, R.J. (2000). Simultaneous Regulation of Hemodynamic and Anesthetic States: A Simulation Study. Annals of Biomedical Engineering, Vol. 28, No. 1, pp. 71-84, ISSN 0090-6964.

Sartori, V., Schumacher, P.M., Bouillon, T., Luginbuehl, M., \& Morari, M. (2005). On-Line Estimation of Propofol Pharmacodynamic Parameters. Proceedings of IEEE Engineering in Medicine and Biology Conference, pp. 74-77, ISBN 0-7803-8740-6, Shanghai, China, September 1-4, 2005.

Schuttler, J., \& Ihmsen, H. (2000). Population Pharmacokinetics of Propofol: A Multicenter Study. Anesthesiology, Vol. 92, No. 3, pp. 727-738, ISSN 1528-1175.

Skogestad, S., \& Postlethwaite, I. (1996). Multivariable Feedback Control Analysis and Design, John Wiley \& Sons, ISBN 978-0-470-01167-6, Chichester, U.K.

Van Poucke, G.E., Brandon Bravo, L.J., \& Shafer, S.L. (2004). Target Controlled Infusions: Targeting the Effect Site While Limiting Peak Plasma Concentration. IEEE Transactions on Biomedical Engineering, Vol. 51, No. 11, pp. 1869-1875, ISSN 00189294.

Varvel, J.R., Donoho, D.L., \& Shafer, S.L. (1992). Measuring the Predictive Performance of Computer-Controlled Infusion Pumps. Journal of Pharmacokinetics and Biopharmaceutics, Vol. 20, No. 1, pp. 63-94, ISSN 0090-466X. 
Zikov, T., Bibian, S., Dumont, G.A., Huzmezan, M., \& Ries, C.R. (2006). Quantifying Cortical Activity During General Anesthesia Using Wavelet Analysis. IEEE Transactions on Biomedical Engineering, Vol. 53, No. 4, pp. 617-632, ISSN 0018-9294. 


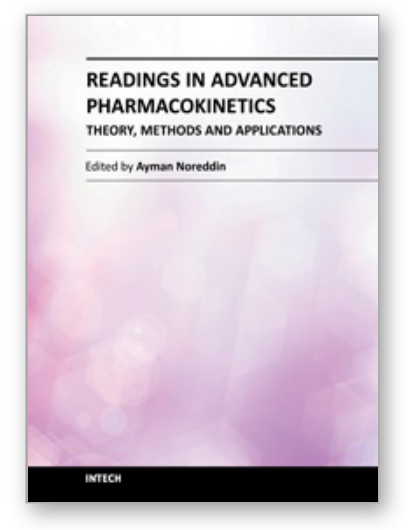

\section{Readings in Advanced Pharmacokinetics - Theory, Methods and Applications}

Edited by Dr. Ayman Noreddin

ISBN 978-953-51-0533-6

Hard cover, 378 pages

Publisher InTech

Published online 20, April, 2012

Published in print edition April, 2012

This book, "Readings in Advanced Pharmacokinetics - Theory, Methods and Applications", covers up to date information and practical topics related to the study of drug pharmacokinetics in humans and in animals. The book is designed to offer scientists, clinicians and researchers a choice to logically build their knowledge in pharmacokinetics from basic concepts to advanced applications. This book is organized into two sections. The first section discusses advanced theories that include a wide range of topics; from bioequivalence studies, pharmacogenomics in relation to pharmacokinetics, computer based simulation concepts to drug interactions of herbal medicines and veterinary pharmacokinetics. The second section advances theory to practice offering several examples of methods and applications in advanced pharmacokinetics.

\section{How to reference}

In order to correctly reference this scholarly work, feel free to copy and paste the following:

Jin-Oh Hahn, Guy A. Dumont and J. Mark Ansermino (2012). Observer-Based Strategies for Anesthesia Drug Concentration Estimation, Readings in Advanced Pharmacokinetics - Theory, Methods and Applications, Dr. Ayman Noreddin (Ed.), ISBN: 978-953-51-0533-6, InTech, Available from:

http://www.intechopen.com/books/readings-in-advanced-pharmacokinetics-theory-methods-andapplications/observer-based-strategies-for-on-line-anesthesia-drug-concentration-estimation

\section{INTECH}

open science | open minds

\author{
InTech Europe \\ University Campus STeP Ri \\ Slavka Krautzeka 83/A \\ 51000 Rijeka, Croatia \\ Phone: +385 (51) 770447 \\ Fax: +385 (51) 686166 \\ www.intechopen.com
}

\author{
InTech China \\ Unit 405, Office Block, Hotel Equatorial Shanghai \\ No.65, Yan An Road (West), Shanghai, 200040, China \\ 中国上海市延安西路65号上海国际贵都大饭店办公楼 405 单元 \\ Phone: +86-21-62489820 \\ Fax: +86-21-62489821
}


(C) 2012 The Author(s). Licensee IntechOpen. This is an open access article distributed under the terms of the Creative Commons Attribution 3.0 License, which permits unrestricted use, distribution, and reproduction in any medium, provided the original work is properly cited. 